\title{
Clinical case report: Unusual and severe psoriatic arthritis mutilans
}

Psoriatic Arthritis (PsA) is considered part of the spondyloarthritis group, which is present in up to $42 \%$ of individuals with psoriasis and up to $15 \%$ of patients with psoriasis may have undiagnosed PsA. Psoriatic arthritis Mutilans is a rare but very aggressive type of advanced joint disease in patients with psoriasis. This report describes the case of a 67 years old patient with family history of psoriasis and undiagnosed psoriatic arthritis with nail lesions for more than 16 years, arthritis for 15 years and severe disease during the last 3 years with disability and bedridden. She had serious joint complications of the disease, as well as severe malnutrition. A missdiagnosis of rheumatoid arthritis was given during 15 years, HLAB27 was positive and radiography of hands and feet showed classic findings of psoriatic arthritis mutilans. We present this case to show the severity of the disease with rapid progression and multiple additional joint complications.

Keywords: psoriatic arthritis $\bullet$ psoriasis $\bullet$ mutilans

\section{Introduction}

The incidence rate of Psoriatic Arthritis (PsA) in patients with psoriasis $(\mathrm{PsO})$ is 1.87 per $100[1,2]$. The clinical manifestations of PsA are complex and heterogeneous. Symptoms of PsA include joint pain and stiffness, skin and nail psoriasis, dactylitis, and persistent, painful enthesitis [3]. Although PsA was once considered a mild disease, it is now understood that progressive structural damage can begin early in the course of PsA [3]. Damage is evidenced by radiologic changes in up to $47 \%$ of patients at a median interval of 2 years of follow-up and it causes irreversible disability [4]. The work of Wright and Moll led to classification of PsA into: polyarticular, asymmetric oligoarticular, distal interphalangeal joint, spondylitis, and arthritis mutilans [5]. We know that the most common presentation, is an overlap between several forms of psoriatic arthritis. PsA may precede skin manifestations by months or years, and in some cases, the changes in the skin may never be present during the course of the disease, making difficult to do the diagnosis [6]. Diagnosis can be based on the family history of psoriasis, genetic markers, presence of dactylitis, enthesitis and pattern of joint affection [6,7]. Despite our growing understanding of the pathogenesis of the disease, there is a delay in the diagnosis of PsA, an entity that is multifactorial and may include an absence of family history, an absence of a duly validated PsA case definition, and an inability to clearly separate seronegative Rheumatoid Arthritis (RA) from PsA [8]. We present a case in which a 67-year-old female patient developed arthritis mutilans in the sight of several doctors with a wrong diagnosis of RA, she did not have skin lesions, but the nail changes were compatible with psoriasis, also she had an inflammatory arthritis disease of 15 years of evolution, which was progressive, severely destructive, polyarticular, symmetric, with axial compromise, that could not fit into any individual category as described by Wright and Moll, until the main pattern of the disease became evident after 15 years of illness and only in management with prednisone and antimalarials, when seen together by dermatology and rheumatology, the diagnosis of Psoriatic Arthritis Mutilans was concluded.

\section{Case presentation}

Initial presentation and history

A 67-year-old woman with a 15-year history of pain in multiple joints and a symmetric and migratory affection. The pain started in low back, associated with morning stiffness for about 2 hours long and gradually affected the knees, ankles, elbows, shoulders, wrists followed by small joints of the hand, metacarpophalangeal joints (MCP), proximal and distal interphalangeal joints (PIP and DIP) and feet. She did not report lesions of any kind on the skin but on nails for more than 10 years. Upon direct questioning about family history about a similar illness, she described desquamative lesions on her father and a spinal condition that left him bedridden to die of complications of an unspecified joint disease, in addition to a daughter with a recent diagnosis of scalp psoriasis The patient had been diagnosed

\section{Alejandra-Lopez $\mathbf{R}^{* 1}$, Karla- Macias $\mathbf{G}^{2}$, Marcela-Cerda $\mathrm{E}^{3}$, Pamela-Sandoval $\mathbf{M}^{3}$, Rossdali-Sanchez $R^{3}$ \& Roxana- Rodriguez $\mathbf{R}^{3}$}

'Internal Medicine doctor and Rheumatologist, Multidisciplinary Model for Psoriasis and Psoriatic Arthritis Clinic PSOAPS co-Director, Principal Investigator in Clinical Trials in CINEA, Guadalajara Jalisco, México

2Dermatologist, Centro Dermatológico del Country Director, Multidisciplinary Model for Psoriasis and Psoriatic Arthritis Clinic PSOAPS co-Director, Principal Investigator in Clinical Trials in CINEA, Guadalajara Jalisco, México

${ }^{3}$ Multidisciplinary Model for Psoriasis and Psoriatic Arthritis PSOAPS Clinic Team, Mexico

*Author for correspondence: dra_alejandralopezrdz@hotmail.com 
with rheumatoid arthritis and treated with NonSteroidal Anti-Inflammatory Drugs (NSAIDs) for joint pain along with some drugs such as hydroxychloroquine and methotrexate taken irregularly due to lack of response to treatment.

\section{Physical examination}

In the physical examination in our multidisciplinary psoriasis clinic, we found a patient in a wheelchair, unable to walk, malnourished and with stable vital signs. The dermatology team reported that the patient had no skin lesions, however, the presence of nail involvement with psoriasis lesions was detected, mainly on the hands and feet, leukonychia, crumbling, onycholysis and hyperkeratosis. She had a sacral pressure ulcer of $8 \mathrm{~cm}$ in diameter with $10 \mathrm{~cm}$ of depth, without infectious characteristics. The rest of the general exam was normal. The Rheumatologist documented the presence of 22 tender and swollen joints count, involving shoulders, elbows, hands, hips, knees and feet. The mobility of the neck was affected with a bilateral cervical rotation of 30 degrees, an impossibility of extension and flexion of the neck, shoulders with pain without possibility of raising the arms, both elbows with contraction in the 20 degree flexion, carpus with hypermobility in all ranges, with hyperflexion, hyperextension, absence of reflexes of the hand, as well as severe atrophy of the muscles. Metacarpophalangeal, proximal and distal interphalangeal joints with hypermobility, telescope fingers with pain and inflammation of most joints. The pain was also present in the hips without mobility, unable to get out of the wheelchair. There was presence of sensitivity (o tenderness??) on both knees associated with mild swelling, as well as pain and swelling in the ankles. Presence of dactylitis of the second toe of the right foot. The range of movements in the spine was severely restricted. Nutritionist reported a severe malnutrition with a body mass index of 15 , it is known as criteria for metabolic syndrome, without diagnosis of diabetes or arterial hypertension. In the diagnostic approach, there were included the next tests: Blood count that showed chronic anemia, blood chemistry with preserved renal function and glucose in $110 \mathrm{mg} / \mathrm{dl}$, liver profile with normal transaminases and albumin in 1.7 g/dl, lipid profile with elevated both LDL and triglycerides. ESR were also requested with a result of $40 \mathrm{~mm} / \mathrm{hr}$ (limit of $20 \mathrm{~mm} / \mathrm{hr}$ ) and CRP of $39 \mathrm{mg} / \mathrm{dl}$ (limit of $6 \mathrm{mg} / \mathrm{dl})$. The rheumatoid factor and anti-CCP were negative. X-rays of the hands revealed the absence of carpal bones bilaterally, with cubitus and continuous radio to the metacarpophalangeal joints without data of ankylosis, a separation of the base of the phalanges with a narrowing of the ends of the metacarpals was also observed. ("pencil in a cup"deformity) (Figures 1 and 2). The distal interphalangeal joints were also involved. A pelvic radiography showed grade IV bilateral sacroiliitis and grade IV bilateral coxarthrosis.

A diagnosis of psoriatic arthritis mutilans was made according to the CASPAR criteria and the definition of Moll and Wright by: digital telescoping, opera glass finger resulting from

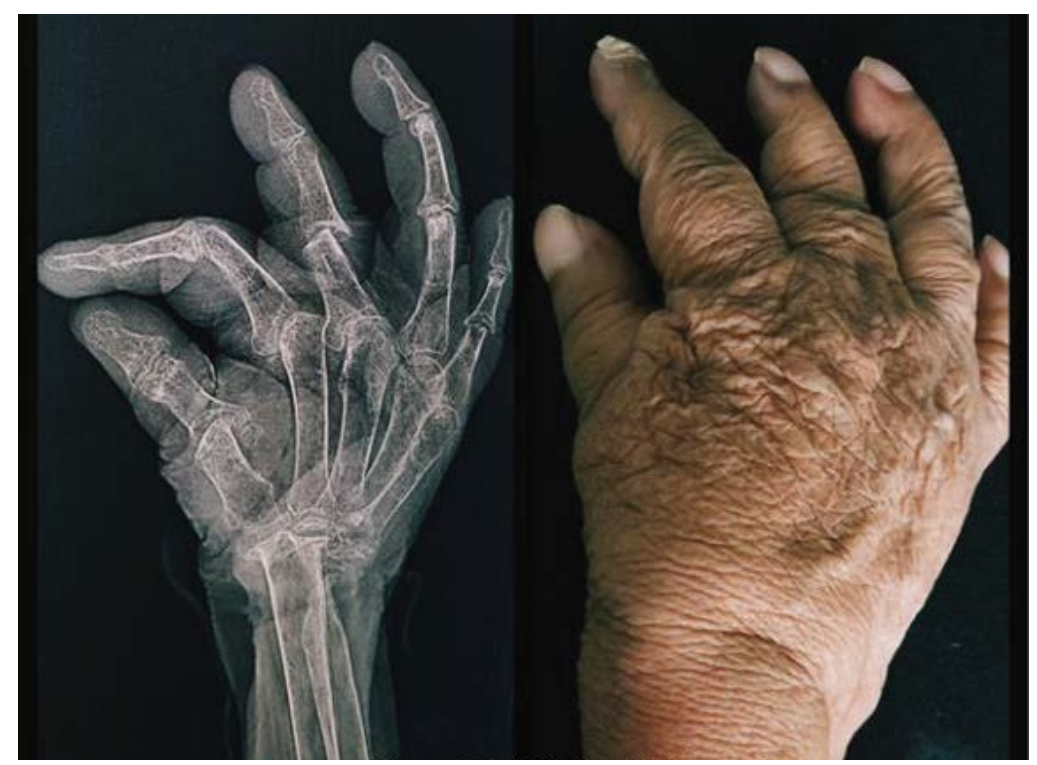

Figure 1. The clinical and radiographic comparison of damage in psoriatic arthritis mutilans, we clinically observed digital telescoping digital shortening and flail joints. 


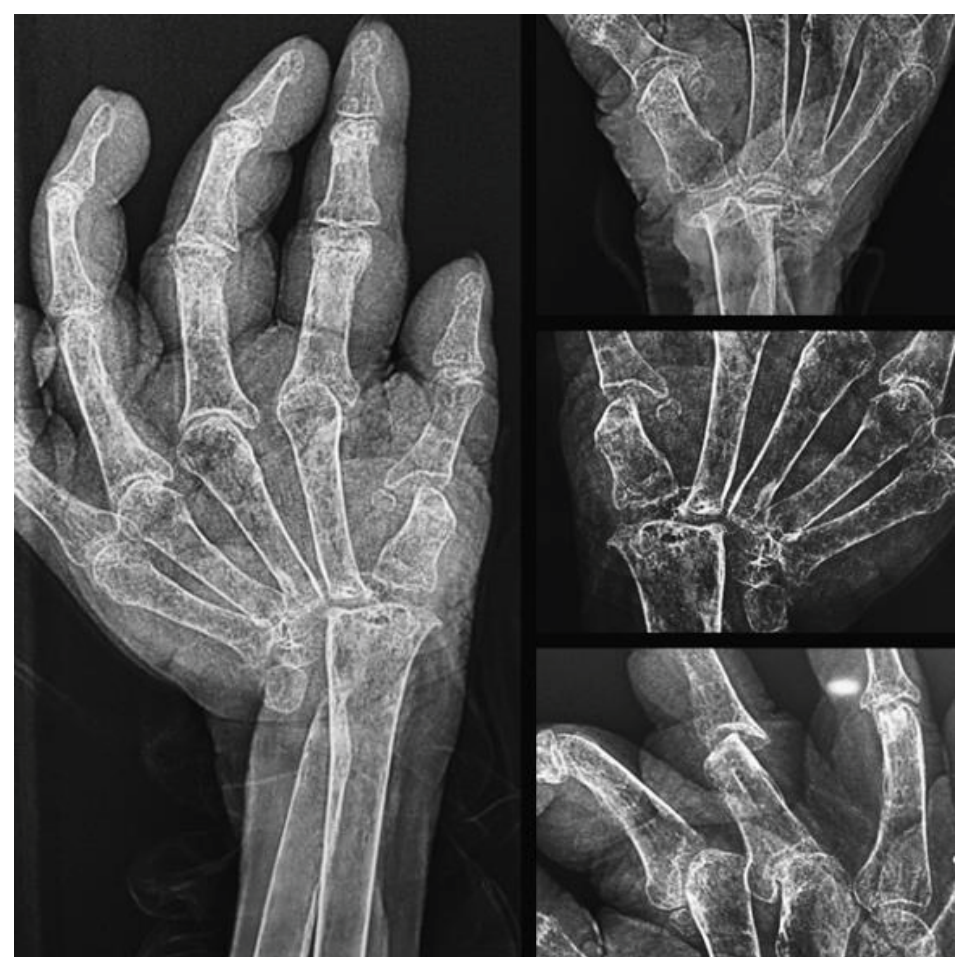

Figure 2. Bone resorption involving the epiphyseal head. Bone resorption extending to the diaphysis. Presence of bone whittling, resorption of bone causing pinpoint end. Presence of pencil-in-cup change, resorption of bone causing cupping of distal or proximal end of the bone with whittling of the opposite side.

PAM: psoriatic arthritis mutilans.

severe osteolysis. The patient's treatment was initiated in a multidisciplinary scheme that included a nutritional plan to improve the malnutrition of the patient and improve the recovery of the sacral ulcer, as well as a physical conditioning plan according to her mobility in order to improve sarcopenia and range of joint mobility. The dermatologist began the treatment of the sacral ulcer with surgery and the debridement of necrotic areas, as well as the hydrocolloid patches and the VAC subsystem to improve the lesion in the sacral area, the rheumatologist decided to start methotrexate $15 \mathrm{mg}$ subcutaneously weekly with $5 \mathrm{mg}$ of folic acid daily in addition to sulfasalazine 500 mg twice a day for 15 days and then $1 \mathrm{~g}$ twice a day. Indomethacin sustained release $75 \mathrm{mg}$ twice daily.

\section{Discussion}

Psoriatic arthritis is now firmly considered as a disease both autoinflammatory and autoimmune, is a chronic, progressive and destructive disease with clear involvement of the IL12 / IL23 axis, and proinflammatory cytokines such as IL23, IL17, TNF as central molecules involved in the pathogenesis [9]. Until a couple of decades ago, arthritis associated with psoriasis was considered a manifestation of rheumatoid arthritis [10,11]. Today we know that they are completely different diseases and we should already talk about a divorce from rheumatoid arthritis of psoriatic arthritis. The global prevalence of PsA is so varied that the ranges vary from $7 \%$ to $50 \%$ depending on the series and the country [12-14], in Mexico there is no data that evidences the epidemiology of psoriatic disease or psoriatic arthritis, less uniform. In up to $20 \%$ of patients with PsA, joint symptoms precede skin manifestations, however, many of these patients have a family history of psoriasis or non-affective conditions [15]. Patients with arthritis before the psoriasis disease tend to be younger, have a positive family history, and are usually men [16].

\section{Conclusion}

Psoriatic arthritis mutilans is a rare but very aggressive type of advanced joint disease in patients with psoriasis. It represents a diagnostic challenge, given the heterogeneity of the manifestations at the beginning of the disease, however, once the damage is established, the distinctive morphology of joint mutilation and disability make the diagnosis late and deficient in this type of disease. Currently, we do not have an effective method to treat psoriatic 
arthritis mutilans, making clear the growing need to better understand the pathophysiology of the disease to develop more effective therapies and stop the rapid progression and potential to destroy the joints, that disables patients permanently. It is evident the importance of the multidisciplinary management of this entity, the clinical conditions of the patient, both acute and chronic, merits the intervention of a nutritionist, but also a psychiatrist, due to the important on mental health of the patient.

\section{Conflicts of interest}

\section{None}

Funding

\section{None}

\section{References}

1. Gladman DD, Antoni C, Mease P et al. Psoriatic arthritis: Epidemiology, clinical features, course, and outcome. Ann. Rheum. Dis. 64 Suppl (2), 14-17 (2005).

2. Eder L, Chandran V, Shen $\mathrm{H}$ et al. Incidence of arthritis in a prospective cohort of psoriasis patients. Arthritis. Care. Res. (Hoboken). 63(4), 619-622 (2011).

3. Mease PJ, Armstrong AW. Managing patients with psoriatic disease: The diagnosis and pharmacologic treatment of psoriatic arthritis in patients with psoriasis. Drugs. 74, 423-441 (2014).

4. Kane D, Stafford L, Bresnihan B et al. A prospective, clinical and radiological study of early psoriatic arthritis: an early synovitis clinic experience. Rheumatology. (Oxford). 42, 14601468 (2003).

5. Moll JMH, Wright V. Psoriatic arthritis. Semin. Arthritis. Rheum. 3, 55-78 (1973).

6. Olivieri I, Padula A, D' Angelo S et al. Psoriatic arthritis sine psoriasis. J. Rheumatol. 83, 28-29 (2009).
7. Gladman DD, Anhorn KA, Schachter RK et al. HLA antigens in psoriatic arthritis. J. Rheumatol. 13(3), 586-592 (1986).

8. Palazzi C, Olivieri I, Petricca A et al. Rheumatoid arthritis or psoriatic symmetric polyarthritis? A difficult differential diagnosis. Clin. Exp. Rheumatol. 20, 3-4 (2002).

9. O' Neill T, Silman AJ. Psoriatic arthritis: historical background and epidemiology. Baillieres. Clin. Rheumatol. 8, 245-261 (1994).

10. Hueber AJ, McInnes IB. Immune regulation in psoriasis and psoriatic arthritis-recent developments. Immunol. Lett. 114(2), 59-65 (2007).

11. Gladman DD. Natural history of psoriatic arthritis. Baillieres. Clin. Rheumatol. 8, 379-394 (1994).

12. Gisondi P, Girolomoni G, Sampogna F et al. Prevalence of psoriatic arthritis and joint complaints in a large population of Italian patients hospitalised for psoriasis. Eur. J. Dermatol. 15, 279-283 (2005).

13. Wilson FC, Icen M, Crowson CS et al. Incidence and clinical predictors of psoriatic arthritis in patients with psoriasis: A population-based study. Arthritis. Rheum. 61(2), 233-239 (2009)

14. Pattison EJ, Harrison BJ, Griffiths CE et al. Environmental risk factors for the development of psoriatic arthritis: results from a case control study. Ann. Rheum. Dis. 67: 672-676 (2008).

15. Reich K, Kruger K, Mossner Ret al. Epidemiology and clinical pattern of psoriatic arthritis in Germany: a prospective interdisciplinary epidemiological study of 1,511 patients with plaque-type psoriasis. Br. J. Dermatol. 160, 1040-1047 (2009).

16. Taylor W, Gladman D, Helliwell $\mathrm{P}$ et al. Classification criteria for psoriatic arthritis: Development of new criteria from a large international study. Arthritis. Rheum. 54, 26652673 (2006). 